\title{
Avaliação de medidas de desempenho para rodovias de pista simples obtidas a partir de relações fluxo-velocidade
}

\author{
José Elievam Bessa Júnior ${ }^{1}$ e José Reynaldo Setti²
}

\begin{abstract}
Resumo: Para estimar o nível de serviço em rodovias de pista simples, o Highway Capacity Manual 2010 (HCM2010) adota como medidas de desempenho a porcentagem de tempo viajando em pelotões (PTSF) e a velocidade média de percurso (ATS). A PTSF, no entanto, é praticamente impossível de ser observada em campo. Na literatura, algumas pesquisas propõem medidas de desempenho alternativas à PTSF que podem ser coletadas diretamente da observação do tráfego. Além disso, as relações fluxo-velocidade têm um papel fundamental na determinação do nível de serviço no HCM2010 e no HBS2001. A meta deste trabalho foi avaliar medidas de desempenho provenientes de relações fluxo-velocidade que pudessem ser adequadas para descrever a qualidade de serviço em rodovias de pista simples no Brasil. Usando dados de tráfego sintéticos produzidos com uma versão do CORSIM calibrada para rodovias no Brasil, foram obtidas relações fluxo-velocidade para diferentes condições (geometria, porcentagens de veículos pesados e velocidades de fluxo livre). Comparações dos valores provenientes desses modelos com dados de campo indicaram que a velocidade média de percurso dos automóveis e a densidade para automóveis poderiam substituir os critérios atuais do HCM para estimar o nível de serviço em rodovias de pista simples no Brasil.
\end{abstract}

Palavras-chave: medidas de desempenho; rodovias de pista simples; relações fluxo-velocidade; simulação de tráfego.

\begin{abstract}
The Highway Capacity Manual 2010 uses Percent-Time-Spent Following (PTSF) and Average Travel Speed to estimate level of service on two-lane rural highways. As it is almost impossible to observe PTSF directly in the field, many researchers have been searching for alternative measures of effectiveness (MDs) that can be obtained from traffic stream parameters. Moreover, speed-flow relationships have a fundamental role in LOS estimation in the HCM2010 and in the German HBS2001. The objective of this paper was to assess MDs derived from speed-flow relationships that could adequately describe quality of service on two-lane rural highways in Brazil. Speed-flow models were fitted for different conditions (geometry, percentages of heavy vehicles and free-flow speeds), using synthetic traffic data produced by a recalibrated version of CORSIM. Comparisons between the values obtained using these models and field data indicated that average travel speed of cars and density for cars could replace the current HCM criteria for the estimation of LOS on two-lane rural highways in Brazil.
\end{abstract}

Keywords: measure of effectiveness; two-lane rural highways; speed-flow relationships; traffic simulation.

\section{INTRODUÇÃO}

O Highway Capacity Manual 2010, ou HCM2010 (TRB, 2010), considera que medidas de desempenho usadas para definirem níveis de serviço em vias devem: (i) refletir a percepção dos usuários ao longo de uma viagem; (ii) ser úteis para os órgãos operacionais; (iii) serem obtidas diretamente da observação em campo; e (iv) ser possíveis de estimá-las baseadas em um modelo para condições específicas do campo. O HCM2010 admite que tanto os critérios como a seleção de medidas de desempenho para determinar o nível de serviço foram feitas sem considerar, adequadamente, a opinião dos usuários, uma vez que pesquisas dessa natureza ainda são incipientes (TRB, 2010, v.1, p. 7-8).

No tocante às rodovias de pista simples, o HCM2010 usa duas medidas de desempenho para determinar o nível de serviço: a velocidade média de percurso $(A T S)$ e a porcentagem de tempo viajando em pelotões $(P T S F)$, sendo essa última praticamente impossível de ser obtida diretamente em campo. Esse fato vai contra as recomendações do

\footnotetext{
1 José Elievam Bessa Júnior, Departamento de Engenharia de

Transportes, CEFET-MG. (elievamjr@gmail.com)

2 José Reynaldo Setti, Departamento de Engenharia de Transportes,

EESC-USP. (jrasetti@usp.br)
}

Manuscrito recebido em 21/04/2016 e aprovado para publicação em 09/09/2016

Este artigo é parte de TRANSPORTES v. 24, n. 3, 2016. ISSN: 2237-1346 (online). DOI:10.4237/transportes.v24i3.1145 próprio manual, o que fez surgir uma linha de pesquisa que busca obter uma medida de desempenho possível de ser obtida diretamente em campo para esse tipo de rodovia. Apesar de haver adaptações locais do HCM em alguns países, como é o caso da Finlândia (Luttinen, 2001), da Argentina (Maldonado et al., 2012) e da Índia (Penmetsa et al., 2015), em alguns países, como a Alemanha e a África do Sul, foram criados métodos próprios para avaliar rodovias de pista simples que dispensam o uso da PTSF (Van As, 2003; FGSV, 2005).

Os principais estudos realizados em rodovias de pista simples no Brasil remetem às adaptações do HCM2000 (TRB, 2000) a partir de dados coletados em estradas paulistas (Egami, 2006; Mon-Ma, 2008). A premissa era de que a estrutura do HCM2000 não deveria ser modificada, mas somente os coeficientes dos modelos de tráfego e as tabelas dos fatores de ajuste. Bessa Jr. e Setti (2011) propuseram outros modelos para as relações entre o fluxo de tráfego e entre a ATS e a PTSF, sem, necessariamente, usar os modelos sugeridos no manual. Uma das conclusões foi a melhor adequação aos dados da relação fluxo-velocidade côncava, em comparação com o modelo linear, usado no HCM2000.

Bessa Jr. e Setti (2012) obtiveram relações fluxo-velocidade côncavas baseadas no manual alemão HBS2001 (FGSV, 2005). Foram obtidas 120 curvas fluxo-velocidade unidirecionais para diferentes alinhamentos vertical e horizontal e porcentagens de veículos pesados. Algumas limitações desse estudo: $(i)$ o alinhamento vertical é determinado 

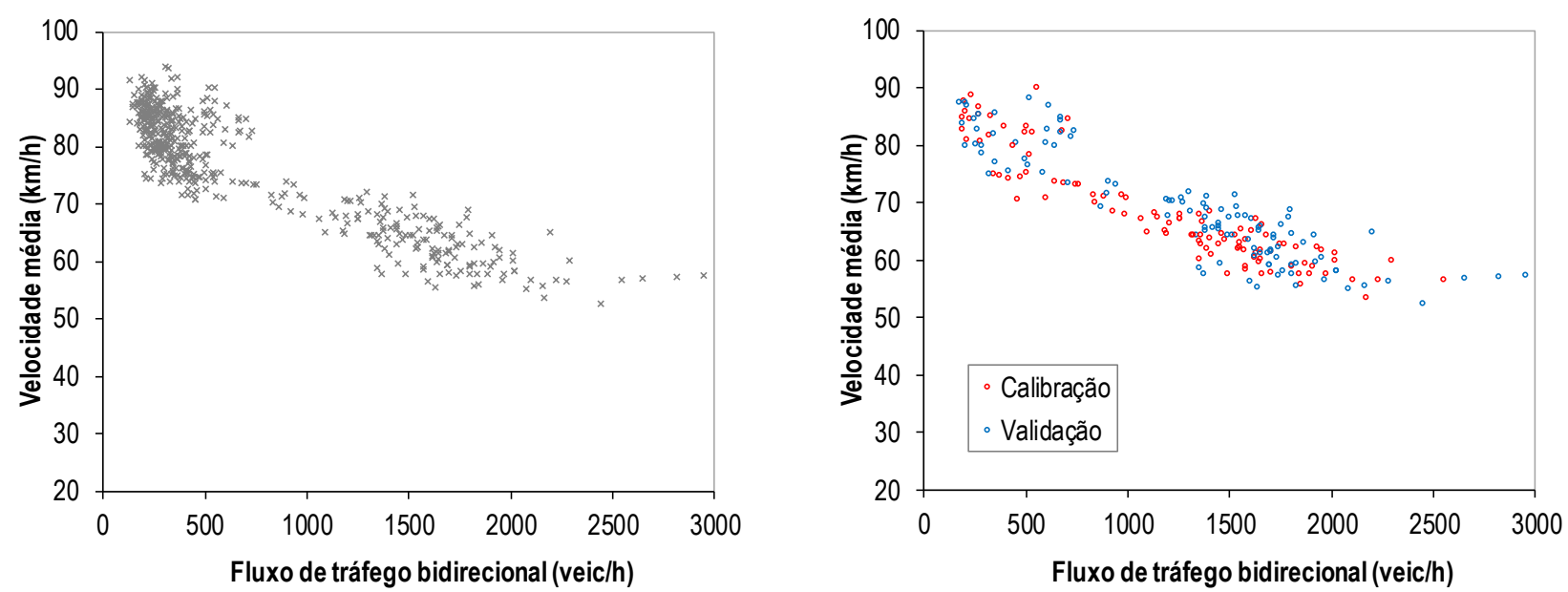

Figura 1. (a) Correntes de tráfego bidirecionais coletadas nas 140 horas de observação; e (b) amostra com 220 observações selecionadas para calibrar e validar o CORSIM

por meio da variável "Classe da Rodovia", que é conceitualmente difícil de ser aplicada e dá resultados inconsistentes para caminhões com desempenho ruim, como os brasileiros; (ii) foi usado um valor médio de $85 \mathrm{~km} / \mathrm{h}$ para as rodovias de pista simples, o que pode dificultar a aplicação dos modelos em rodovias com diferentes velocidades de fluxo de livre; e (iii) os cenários estudados limitam-se a porcentagens de veículos pesados inferiores a $25 \%$, o que também pode reduzir a utilidade das curvas obtidas.

Bessa Jr. e Setti (2013) avaliaram a possibilidade de estimar a PTSF a partir de modelos que usam dados de tráfego facilmente observados em campo. Os modelos testados para estimar a PTSF incluíam, além da porcentagem de veículos em pelotões, (sugerida pelo HCM como medida proxy da $P T S F$ ), três outros modelos encontrados na literatura (Pursula, 1995; Cohen e Polus, 2011; Laval, 2006) e uma quinta proposta que consistia num modelo multilinear. Nenhum dos modelos forneceu resultados confiáveis, pelo menos para os dados usados no teste, que foram coletados em rodovias do estado de São Paulo.

É possível perceber a necessidade de se encontrar medidas de desempenho que possam ser diretamente coletadas em campo para substituir a PTSF como critério para determinação do nível de serviço. Com base nisso, a meta deste trabalho foi avaliar a adequação de medidas de desempenho que podem ser obtidas a partir da relação fluxo-velocidade para avaliar a qualidade de serviço em rodovias de pista simples brasileiras. As medidas de desempenho escolhidas para isso referem-se a um sentido da corrente de tráfego e são: $(i)$ a densidade dos automóveis na corrente de tráfego $\left(D_{d, c a r}\right)$, que é usada no manual alemão de capacidade (FGSV, 2005); (ii) o atraso percentual dos automóveis, $P D_{d, \text { car }}$ (Yu e Washburn, 2009); e (iii) a velocidade média de percurso dos automóveis $\left(A T S_{d, c a r}\right)$.

\section{COLETA DE DADOS E CALIBRAÇÃO DO CORSIM}

Os dados de tráfego foram usados com dois propósitos: $(i)$ calibrar e validar o simulador escolhido, o CORSIM (McTrans, 2010); e (ii) avaliar a adequabilidade dos modelos de tráfego desenvolvidos. Para obter os dados de tráfego usados nesta pesquisa, foram usados os seguintes métodos: (i) filmagem da corrente de tráfego; (ii) estações permanentes de monitoramento de tráfego com sensores instalados no pavimento; e (iii) dados coletados a partir do uso de GPS.

O método de obtenção dos dados por meio de filmagem da corrente consiste em instalar câmeras de vídeo nas extremidades e em pontos intermediários do trecho. Os dados de tráfego são obtidos da observação posterior das filmagens e podem incluir uma ampla gama de dados, como velocidades de percurso, headways, tipo do veículo, entre outros. O conjunto de dados de tráfego coletados com filmagem foi obtido em 15 trechos de rodovias de pista simples paulistas em pesquisas anteriores (Egami, 2006, MonMa, 2008, Bessa Jr. e Setti, 2011; Bessa Jr. e Setti, 2013), principalmente para calibrar e validar simuladores de tráfego. Para este artigo, este conjunto de informações foi ampliado com coletas realizadas em um outro trecho, a SPA110/300, entre o $\mathrm{km} \mathrm{5,0} \mathrm{e} \mathrm{o} \mathrm{km} \mathrm{6,5,} \mathrm{que} \mathrm{liga} \mathrm{a} \mathrm{Rodovia}$ Anhanguera a Paulínia, SP, escolhido pelos períodos com grande fluxo veicular. Nos 16 trechos de rodovias, foram realizadas 140 horas de filmagens.

A Figura 1(a) mostra os dados velocidade-fluxo coletados através das filmagens. É possível perceber que uma grande quantidade de dados foi coletada em períodos com fluxo baixo, de até $500 \mathrm{veic} / \mathrm{h}$. Por essa razão, obteve-se uma amostra deste conjunto de dados de tal forma que as observações estivessem bem distribuídas ao longo da faixa de fluxos observada, para evitar distorções causadas pelo maior número de observações com fluxos baixos. A Figura 1(b) mostra as 220 correntes de tráfego selecionadas para calibrar e validar o CORSIM (Bessa Jr., 2015).

Os dados obtidos das filmagens foram usados para calibrar os parâmetros comportamentais do CORSIM. Esse método de coleta é mais confiável que uma coleta a partir de sensores de tráfego, que obtém informações em um ponto da rodovia e, por essa razão, às vezes podem não ser representativos para um trecho; além disso, ainda podem ter inconsistências, como confundir dois veículos muito próximos com um veículo longo. Assim, os dados provenientes das filmagens foram os únicos dados usados para validar os modelos de tráfego desenvolvidos, como pode ser visto mais adiante. O método de calibração e validação do CORSIM é fortemente baseado no proposto em um estudo anterior (Bessa Jr. e Setti, 2012) e foi realizado a partir de um Algoritmo Genético (AG). O modelo calibrado representa 

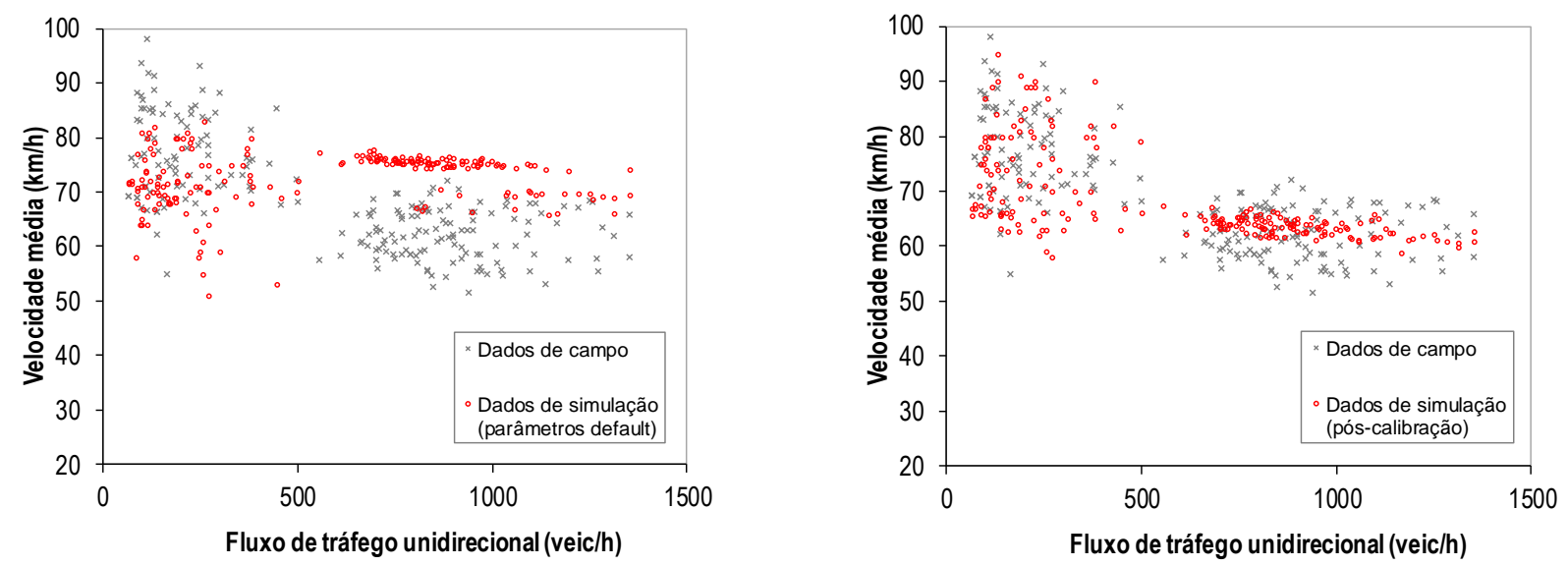

Figura 2. (a) Comparação entre correntes de tráfego observadas no campo e simuladas pelo CORSIM antes da calibração; e (b) comparação dos dados obtidos em campo com as correntes simuladas com a versão recalibrada do CORSIM

uma "rodovia média", na qual o comportamento do tráfego reflete o que foi observado nas estradas estudadas.

Para aplicação do AG, foi escolhida uma função fitness que consiste na média dos coeficientes de correlação entre as frequências dos histogramas dos dados de campo e simulados com o CORSIM (velocidades médias de viagem e headways nos pontos de observação, ambos por sentido). Usando-se os valores default fornecidos pelo CORSIM para os parâmetros de calibração, obteve-se um valor para a função fitness igual a 0,64. Com a implementação do AG, a melhor solução encontrada proporcionou uma sensível melhora nos resultados, medidos pela função fitness cujo valor foi $25 \%$ maior se comparado ao produzido pela simulação que usa valores default para os parâmetros de calibração. Quando o melhor resultado da calibração foi aplicado para o conjunto de dados da validação, verificou-se um aumento de $22 \%$ no valor do fitness em relação ao valor da função fitness encontrado usando-se a versão não calibrada do CORSIM.

Os dados provenientes de sensores de tráfego foram usados para: $(i)$ planejar coletas de campo com as filmadoras; (ii) determinar a velocidade de fluxo livre direcional dos segmentos para modelagem no CORSIM; e (iii) estimar o headway médio mínimo, um input do simulador. Foram analisadas as informações de 44 estações permanentes de monitoramento de tráfego, sendo 17 gerenciadas pela concessionária responsável pela MG-50, 4 pelo DER-SP e 23 por concessionárias paulistas.

O CORSIM foi calibrado em duas etapas: uma para ajuste dos parâmetros do modelo de desempenho veicular; e uma outra para calibrar as lógicas de car-following, de ultrapassagens e de velocidades desejadas. Em cada etapa, foi conduzido um experimento distinto. Maiores detalhes sobre os parâmetros ajustados em cada submodelo do CORSIM podem ser obtidos em Bessa Jr. (2015).

Os parâmetros de calibração do modelo de desempenho veicular estão armazenados no Record Type 173 do CORSIM. Para calibrá-los, foram coletados dados da massa e da potência dos caminhões em um posto de pesagem na Rodovia Washington Luís (SP-310, km 200) em 2011. Perfis de velocidade ao longo de um segmento de $11 \mathrm{~km}$ foram coletados a intervalos de 1,0 $\mathrm{s}$ através de um GPS instalado em caminhões que passavam pela balança. A calibração também foi realizada por meio de um $\mathrm{AG}$, com caracterís- ticas semelhantes ao desenvolvido num estudo anterior $(\mathrm{Cu}-$ nha et al., 2009). A função fitness usada é a diferença média entre as curvas de desempenho (velocidade dos caminhões versus distância nas rampas ascendentes) simuladas e observadas. A aplicação do AG proporcionou uma melhoria na função fitness da ordem de $60 \%$ em relação aos resultados obtidos sem calibrar o simulador.

A Figura 2 mostra como a versão recalibrada do CORSIM fornece melhores resultados do que a versão original (sem calibração). Para o conjunto de dados usados na calibração, a figura mostra os pontos fluxo-velocidade unidirecionais obtidos antes e após calibração pelo AG, em comparação com os dados de campo.

\section{AVALIAÇÃO DAS MEDIDAS DE DESEMPENHO}

Nesta pesquisa, foi examinada a adequação de duas medidas de desempenho que podem ser estimadas diretamente da relação fluxo-velocidade. A primeira delas é a usada pelo HBS2001 para avaliar a qualidade de serviço em rodovias de pista simples, a densidade dos automóveis $\left(D_{d, c a r}\right)$ :

$$
D_{d, c a r}=\frac{q_{d}}{A T S_{d, c a r}}
$$

em que $q_{d}$ é o fluxo de tráfego direcional (veic/h) e $A T S_{d, c a r}$ é a velocidade média de percurso dos automóveis na direção analisada. O fluxo de tráfego é facilmente observável e a velocidade percurso é obtida da relação entre fluxo e $A T S_{\text {d,car }}$.

A segunda medida examinada é o atraso percentual $\left(P D_{d, c a r}\right)$, proposto por Yu e Washburn (2009), que possui a seguinte formulação:

$$
P D_{d, c a r}=\frac{A T T_{d, c a r}-A T T_{F F S_{d}}}{A T T_{F F S_{d}}} \times 100
$$

sendo $A T S_{d, \text { car }}$ a velocidade média de percurso dos automóveis na direção analisada $(\mathrm{km} / \mathrm{h}) ; A T T_{d, c a r}$ o tempo médio de viagem dos automóveis na direção analisada (s); e $A T T_{F F S d}$, o tempo médio de viagem se a velocidade média de percurso fosse igual à $F F S_{d}$ na direção analisada (s). 


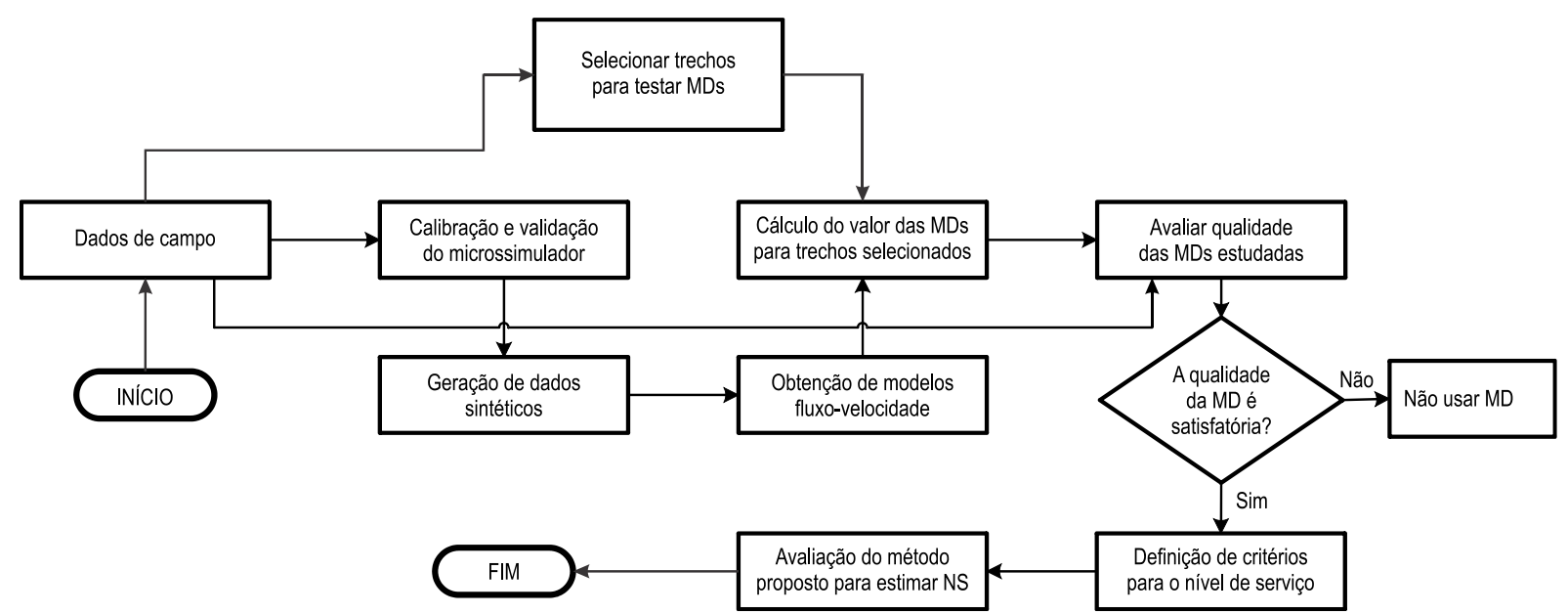

Figura 3. Método proposto para avaliação de medidas de desempenho capazes de indicar a qualidade de serviço em rodovias de pista simples

\subsection{Método usado para avaliar as medidas de desempenho}

O método proposto para análise de medidas de desempenho (MDs) para rodovias de pista simples é apresentado na Figura 3. O processo inicia-se com a coleta de dados de tráfego nos trechos escolhidos. Esses dados são usados para calibrar e validar a calibração do simulador escolhido (no caso, CORSIM). Uma vez que o simulador calibrado seja considerado capaz de reproduzir o comportamento das correntes de tráfego observadas nas rodovias escolhidas, gera-se o conjunto de dados de tráfego sintéticos que será usado para a criação dos modelos. Os dados sintéticos são necessários porque é absolutamente inviável observar diretamente no campo correntes de tráfego com toda a gama de fluxos e porcentagens de caminhões necessária para a criação dos modelos, para as características de projeto geométrico e velocidade de fluxo livre desejadas (Bessa Jr. e Setti, 2011).

Com os modelos que expressam as relações velocidade-fluxo, são estimados os valores das medidas de desempenho para um conjunto de trechos selecionados, para os quais estão disponíveis filmagens da corrente de tráfego. A adequação da medida de desempenho é verificada por meio de funções de qualidade de ajustamento, que comparam os valores estimados pelo modelo com os valores observados em campo para cada medida de desempenho testada. Essa comparação determina quais medidas de desempenho poderiam ser ou não usadas para criar um critério de nível de serviço.

Caso a medida de desempenho esteja apta a ser usada, é preciso determinar quais serão os critérios para definir os níveis de serviço. Por fim, deve ser avaliado se o método proposto subestima ou superestima o nível de serviço em comparação com o método do HCM, que tem sido amplamente recomendado pelos órgãos brasileiros que gerenciam o tráfego em rodovias.

\subsection{Geometria das vias}

Tanto nos manuais HCM e HBS quanto em estudos brasileiros (Egami, 2006; Mon-Ma, 2008; Bessa Jr. e Setti, 2011; Bessa Jr. e Setti, 2012), modelos de tráfego foram desenvolvidos com dados produzidos por simulação em segmentos hipotéticos, cuja geometria dificilmente é encon- trada numa rodovia real. A fim de evitar esse problema, decidiu-se usar o projeto geométrico dos trechos em que estavam instaladas as estações de monitoramento de tráfego, cujos dados foram disponibilizados para este estudo, para gerar os dados sintéticos e os modelos de tráfego desta pesquisa. Com isso, visava-se produzir modelos de tráfego que se adequassem melhor às condições do campo do que modelos obtidos com segmentos hipotéticos. Assim, foram delimitados segmentos homogêneos de até $10 \mathrm{~km}$ de extensão (dentro dos quais estavam os laços indutivos) para serem simulados no CORSIM. Para evitar distorções nos modelos, nessa amostra não foram incluídos aqueles segmentos onde foram coletados dados através de filmagens, já que esses trechos forneceram os dados para calibrar e validar o CORSIM.

Para representar o alinhamento vertical dos segmentos homogêneos, foi utilizado o parâmetro rise and fall $(R F)$, definido como o somatório da altitude de aclives e declives, em metros, de um determinado segmento com comprimento em quilômetros (Kerali et al., 2000). Segmentos com $R F$ abaixo de $20 \mathrm{~m} / \mathrm{km}$ representam relevos planos, enquanto segmentos com $R F$ em torno de $80 \mathrm{~m} / \mathrm{km}$ representam segmentos com relevo montanhoso. Com base nisso, foram estabelecidas as seguintes classes para os alinhamentos verticais: $0 \leq R F \leq 20 \mathrm{~m} / \mathrm{km} ; 20<R F \leq 40$ $\mathrm{m} / \mathrm{km} ; 40<R F \leq 60 \mathrm{~m} / \mathrm{km} ; 60<R F \leq 80 \mathrm{~m} / \mathrm{km}$; e $R F>80$ $\mathrm{m} / \mathrm{km}$.

No Brasil, Nunes et al. (2010) usaram o $R F$ para analisar, economicamente, a influência do alinhamento vertical nos custos de construção de rodovias. Andrade e Setti (2011) basearam-se nesse parâmetro para caracterizar segmentos homogêneos de rodovias de pista dupla paulistas. $\mathrm{O}$ Manual de Gerência de Pavimentos do DNIT (DNIT, 2011) utiliza o Sistema HDM-4 (Highway Development and Management System) para realizar as avaliações econômicas necessárias para utilização do Sistema de Gerência de Pavimentos do DNIT (SGP-DNIT). O parâmetro $R F$ consiste numa das informações necessárias para utilizar o HDM-4. Assim, buscando-se propor um método simples e que possa ser compatibilizado com outros métodos para avaliar rodovias brasileiras, a utilização do $R F$ parece ser razoável.

Outro parâmetro que é usado pelo HDM-4 e, consequentemente, pelo SGP-DNIT, é a curvatura $K U$, definido como o somatório das mudanças angulares no alinhamento horizontal, dado em graus, em um determinado segmento de rodovia, com comprimento dado em quilômetros. 
Neste trabalho, foram considerados cinco categorias do alinhamento horizontal: $0 \leq K U \leq 50^{\circ} / \mathrm{km} ; 50<K U \leq$ $100 \% / \mathrm{km} ; 100<K U \leq 150^{\circ} / \mathrm{km} ; 150<K U \leq 200^{\circ} / \mathrm{km} ; \mathrm{e}$ $K U>200 \% \mathrm{~km}$.

Com cinco categorias para o alinhamento vertical e cinco categorias para o alinhamento horizontal, é possível dizer que, do ponto de vista da geometria, podem existir 25 tipos de segmentos homogêneos. Os 44 segmentos no entorno dos sensores que poderiam ser usados para gerar dados sintéticos tiveram seus valores de $R F$ e $K U$ calculados e classificados como um desses 25 tipos. Entretanto, para que os modelos pudessem ser desenvolvidos e testados, decidiu-se que apenas seriam considerados os tipos de segmentos que contemplassem as coletas de dados através de filmagem.

Além disso, decidiu-se também excluir dessa amostra os trechos que foram usados para a calibração do CORSIM, já que o simulador foi especificamente calibrado usando os dados dessas filmagens. Dessa forma, a amostra continha 22 segmentos homogêneos em quatro combinações de $R F$ e $K U$, que estão mostradas na Tabela 1.

Tabela 1. Classificação dos segmentos homogêneos usados para gerar dados sintéticos para os modelos

\begin{tabular}{lcc}
\hline$K U$ & $R F(\mathrm{~m} / \mathrm{km})$ & \\
\cline { 2 - 3 }$(\% / \mathrm{km})$ & $0-20$ & $20-40$ \\
\hline $0-50$ & 5 & 15 \\
$100-150$ & - & 1 \\
$150-200$ & 1 & - \\
\hline
\end{tabular}

\subsection{Modelos de tráfego}

Foram avaliados dois modelos para a relação fluxovelocidade: um modelo linear, utilizado no HCM2010 (Equação 3), e um modelo côncavo, que é usado pelo HBS2001 (Equação 4). Seguindo a tendência do HCM e do HBS, foram obtidos somente modelos direcionais sem considerar a influência do fluxo oposto no sentido analisado, cuja forma geral é:

$$
\begin{aligned}
& A T S_{d, c a r}=a+b \cdot q_{d}, \\
& A T S_{d, c a r}=a+b \cdot \sqrt{q_{d}},
\end{aligned}
$$

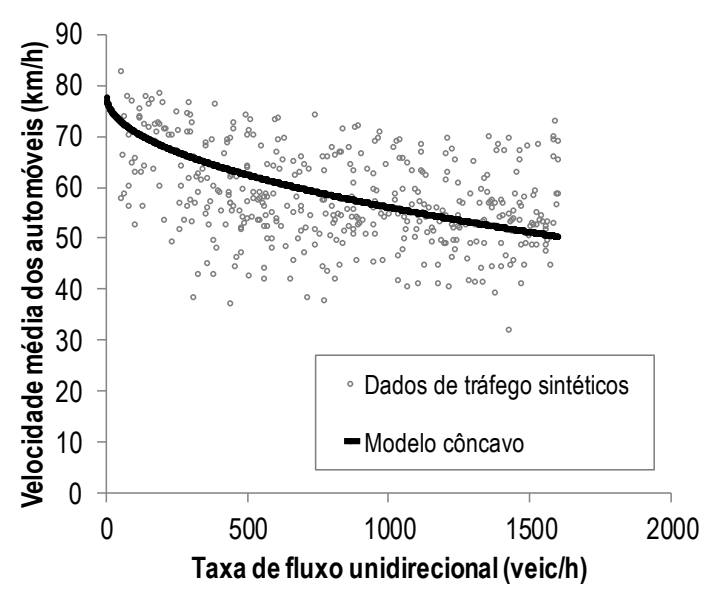

(a) Côncava $\left(R^{2}=0,84\right)$

Figura 4. Relações fluxo-velocidade obtidas para RF $\leq 20 \mathrm{~m} / \mathrm{km}, \mathrm{KU} \leq 50^{\circ} / \mathrm{km}, \mathrm{Phv}=0 \%$ e FFS $=80 \mathrm{~km} / \mathrm{h}$ em que $A T S_{d, c a r}$ é a velocidade média de percurso direcional dos automóveis $(\mathrm{km} / \mathrm{h})$; $q_{d}$ é o fluxo de tráfego no sentido analisado (veic/h); e $a$ e $b$ são parâmetros obtidos por regressão. Os modelos foram ajustados a partir de dados sintéticos, produzidos a partir de simulações que recriam as condições desejadas de fluxo de tráfego, porcentagem de caminhões, geometria do trecho e velocidade de fluxo livre na direção desejada, conforme método usado num estudo anterior (Bessa Jr. e Setti, 2011).

Para uma dada velocidade de fluxo livre direcional $\left(F F S_{d}\right)$, foram desenvolvidas 24 funções relacionando o fluxo e a velocidade média de percurso, resultantes da combinação de quatro valores entre os parâmetros $R F$ e $K U$ (Tabela 1 ) e seis valores para a porcentagem de veículos pesa$\operatorname{dos} P_{h v}$ (de 0 a $50 \%$, em intervalos de $10 \%$ ). Foram obtidos modelos para quatro valores de $F F S_{d}(70,80,90$ e 100 $\mathrm{km} / \mathrm{h}$ ), resultando em $4 \times 6 \times 4=96$ relações fluxo-velocidade. Para que houvesse 500 correntes de tráfego na regressão de cada modelo, foram simuladas $500 \times 96=48.000$ cenários, replicados cinco vezes cada. Os modelos foram gerados sem faixas adicionais e com as zonas de ultrapassagens observadas em campo. As Tabelas 2 e 3 mostram exemplos de parâmetros $a$ e $b$ obtidos para os modelos linear e côncavo com $F F S_{d}$ igual a $70 \mathrm{~km} / \mathrm{h}$. Os demais parâmetros podem ser obtidos em Bessa Jr. (2015).

Das Tabelas 2 e 3 pode-se notar que $R^{2}$ é sistematicamente maior para o modelo côncavo do que para o modelo linear; no entanto, a diferença é marginal, sugerindo que o modelo linear também é razoável. O mesmo comportamento de $R^{2}$ foi encontrado para os modelos com $F F S_{d}$ iguais a 80,90 e $100 \mathrm{~km} / \mathrm{h}$. Para efeito de comparação com dados de campo em seções subsequentes, foi escolhido o modelo côncavo para a relação fluxo-velocidade. A Figura 4 mostra um exemplo dos modelos obtidos por regressão a partir de dados sintéticos gerados com o CORSIM, para $R F$ $\leq 20 \mathrm{~m} / \mathrm{km}, K U \leq 50^{\circ} / \mathrm{km}, P_{h v}=0 \%$ e $F F S=80 \mathrm{~km} / \mathrm{h}$. Percebe-se que o modelo côncavo se ajusta melhor aos dados, o que explica o maior coeficiente $R^{2}$ obtido em relação ao modelo linear.

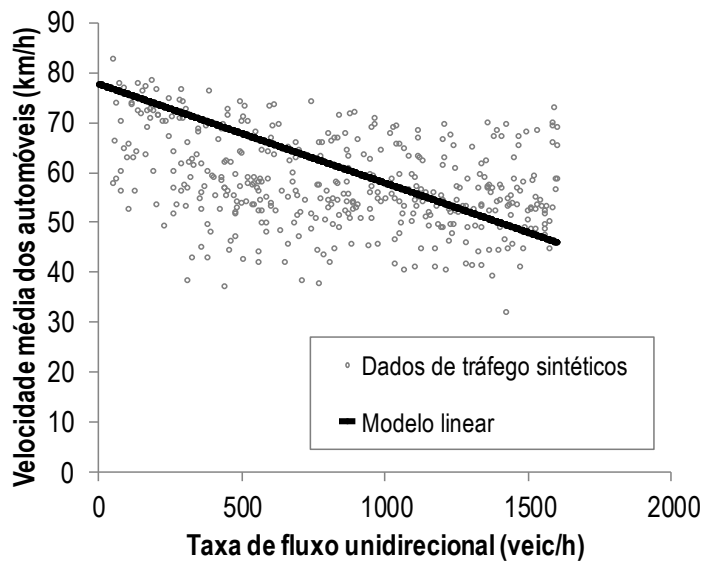

(b) Linear $\left(R^{2}=0,76\right)$ 
Tabela 2. Parâmetros do modelo linear da relação fluxo-velocidade com $F F S d=70 \mathrm{~km} / \mathrm{h}$

\begin{tabular}{|c|c|c|c|c|c|c|c|c|c|c|c|c|c|}
\hline \multicolumn{7}{|c|}{$P_{h v}=0 \%$} & \multicolumn{7}{|c|}{$P_{h v}=30 \%$} \\
\hline \multirow{3}{*}{$\begin{array}{c}K U \\
(\text { graus } / \mathrm{km})\end{array}$} & \multicolumn{6}{|c|}{$R F(\mathrm{~m} / \mathrm{km})$} & \multirow{3}{*}{$\begin{array}{c}K U \\
\text { (graus/km) }\end{array}$} & \multicolumn{6}{|c|}{$R F(\mathrm{~m} / \mathrm{km})$} \\
\hline & \multicolumn{3}{|c|}{$\leq 20$} & \multicolumn{3}{|c|}{$20-40$} & & \multicolumn{3}{|c|}{$\leq 20$} & \multicolumn{3}{|c|}{$20-40$} \\
\hline & $a$ & $b$ & $R^{2}$ & $a$ & $b$ & $R^{2}$ & & $a$ & $b$ & $R^{2}$ & $a$ & $b$ & $R^{2}$ \\
\hline$\leq 50$ & 64,61 & $-0,015$ & 0,73 & 67,26 & $-0,017$ & 0,77 & $\leq 50$ & 68,11 & $-0,020$ & 0,78 & 62,14 & $-0,019$ & 0,77 \\
\hline $100-150$ & - & - & - & 58,67 & $-0,014$ & 0,76 & $100-150$ & - & - & - & 54,53 & $-0,028$ & 0,69 \\
\hline $150-200$ & 57,91 & $-0,012$ & 0,65 & - & - & - & $150-200$ & 59,60 & $-0,020$ & 0,82 & - & - & - \\
\hline \multicolumn{7}{|c|}{$P_{h v}=10 \%$} & \multicolumn{7}{|c|}{$P_{h v}=40 \%$} \\
\hline \multirow{3}{*}{$\begin{array}{c}K U \\
\text { (graus/km) }\end{array}$} & \multicolumn{6}{|c|}{$R F(\mathrm{~m} / \mathrm{km})$} & & \multicolumn{6}{|c|}{$R F(\mathrm{~m} / \mathrm{km})$} \\
\hline & \multicolumn{3}{|c|}{$\leq 20$} & \multicolumn{3}{|c|}{$20-40$} & $\begin{array}{c}K U \\
\text { (graus } / \mathrm{km})\end{array}$ & \multicolumn{3}{|c|}{$\leq 20$} & \multicolumn{3}{|c|}{$20-40$} \\
\hline & $a$ & $b$ & $R^{2}$ & $a$ & $b$ & $R^{2}$ & & $a$ & $b$ & $R^{2}$ & $a$ & $b$ & $R^{2}$ \\
\hline$\leq 50$ & 66,60 & $-0,017$ & 0,75 & 68,16 & $-0,023$ & 0,77 & $\leq 50$ & 67,30 & $-0,018$ & 0,77 & 61,97 & $-0,021$ & 0,74 \\
\hline $100-150$ & - & - & - & 57,11 & $-0,026$ & 0,71 & $100-150$ & - & - & - & 54,58 & $-0,030$ & 0,80 \\
\hline $150-200$ & 57,96 & $-0,015$ & 0,74 & - & - & - & $150-200$ & 61,57 & $-0,022$ & 0,85 & - & - & - \\
\hline \multicolumn{7}{|c|}{$P_{h v}=20 \%$} & \multicolumn{7}{|c|}{$P_{h v}=50 \%$} \\
\hline \multirow{3}{*}{$\begin{array}{c}K U \\
(\text { graus/km) }\end{array}$} & \multicolumn{6}{|c|}{$R F(\mathrm{~m} / \mathrm{km})$} & & \multicolumn{6}{|c|}{$R F(\mathrm{~m} / \mathrm{km})$} \\
\hline & \multicolumn{3}{|c|}{$\leq 20$} & \multicolumn{3}{|c|}{$20-40$} & $\begin{array}{c}K U \\
\text { (graus/km) }\end{array}$ & \multicolumn{3}{|c|}{$\leq 20$} & \multicolumn{3}{|c|}{$20-40$} \\
\hline & $a$ & $b$ & $R^{2}$ & $a$ & $b$ & $R^{2}$ & & $a$ & $b$ & $R^{2}$ & $a$ & $b$ & $R^{2}$ \\
\hline$\leq 50$ & 64,05 & $-0,015$ & 0,73 & 69,36 & $-0,026$ & 0,78 & $\leq 50$ & 63,60 & $-0,016$ & 0,71 & 62,30 & $-0,023$ & 0,76 \\
\hline $100-150$ & - & - & - & 57,91 & $-0,033$ & 0,77 & $100-150$ & - & - & - & 57,55 & $-0,032$ & 0,85 \\
\hline $150-200$ & 57,18 & $-0,015$ & 0,68 & - & - & - & $150-200$ & 58,86 & $-0,020$ & 0,85 & - & - & - \\
\hline
\end{tabular}

Tabela 3. Parâmetros do modelo côncavo da relação fluxo-velocidade com $F F S d=70 \mathrm{~km} / \mathrm{h}$

\begin{tabular}{|c|c|c|c|c|c|c|}
\hline \multicolumn{7}{|c|}{$P_{h v}=0 \%$} \\
\hline \multirow{3}{*}{$\begin{array}{c}K U \\
(\text { graus} / \mathrm{km})\end{array}$} & \multicolumn{6}{|c|}{$R F(\mathrm{~m} / \mathrm{km})$} \\
\hline & \multicolumn{3}{|c|}{$\leq 20$} & \multicolumn{3}{|c|}{$20-40$} \\
\hline & $a$ & $b$ & $R^{2}$ & $a$ & $b$ & $R^{2}$ \\
\hline$\leq 50$ & 64,61 & $-0,520$ & 0,80 & 67,26 & $-0,587$ & 0,84 \\
\hline $100-150$ & - & - & - & 58,67 & $-0,488$ & 0,83 \\
\hline $150-200$ & 57,91 & $-0,391$ & 0,71 & - & - & - \\
\hline \multicolumn{7}{|c|}{$P_{h v}=10 \%$} \\
\hline \multirow{3}{*}{$\begin{array}{c}K U \\
(\text { graus/km) }\end{array}$} & \multicolumn{6}{|c|}{$R F(\mathrm{~m} / \mathrm{km})$} \\
\hline & \multicolumn{3}{|c|}{$\leq 20$} & \multicolumn{3}{|c|}{$20-40$} \\
\hline & $a$ & $b$ & $R^{2}$ & $a$ & $b$ & $R^{2}$ \\
\hline$\leq 50$ & 66,60 & $-0,580$ & 0,83 & 68,16 & $-0,746$ & 0,86 \\
\hline $100-150$ & - & - & - & 57,11 & $-0,637$ & 0,79 \\
\hline $150-200$ & 57,96 & $-0,481$ & 0,82 & - & - & - \\
\hline
\end{tabular}

\begin{tabular}{|c|c|c|c|c|c|c|}
\hline \multicolumn{7}{|c|}{$P_{h v}=30 \%$} \\
\hline \multirow{3}{*}{$\begin{array}{c}K U \\
\text { (graus/km) }\end{array}$} & \multicolumn{6}{|c|}{$R F(\mathrm{~m} / \mathrm{km})$} \\
\hline & \multicolumn{3}{|c|}{$\leq 20$} & \multicolumn{3}{|c|}{$20-40$} \\
\hline & $a$ & $b$ & $R^{2}$ & $a$ & $b$ & $R^{2}$ \\
\hline$\leq 50$ & 68,11 & $-0,668$ & 0,86 & 62,14 & $-0,613$ & 0,83 \\
\hline $100-150$ & - & - & - & 54,53 & $-0,707$ & 0,80 \\
\hline $150-200$ & 59,60 & $-0,649$ & 0,87 & - & - & - \\
\hline \multicolumn{7}{|c|}{$P_{h v}=40 \%$} \\
\hline \multirow{3}{*}{$\begin{array}{c}K U \\
\text { (graus/km) }\end{array}$} & \multicolumn{6}{|c|}{$R F(\mathrm{~m} / \mathrm{km})$} \\
\hline & \multicolumn{3}{|c|}{$\leq 20$} & \multicolumn{3}{|c|}{$20-40$} \\
\hline & $a$ & $b$ & $R^{2}$ & $a$ & $b$ & $R^{2}$ \\
\hline$\leq 50$ & 67,30 & $-0,608$ & 0,84 & 61,97 & $-0,629$ & 0,81 \\
\hline $100-150$ & - & - & - & 54,58 & $-0,763$ & 0,88 \\
\hline $150-200$ & 61,57 & $-0,683$ & 0,90 & - & - & - \\
\hline \multicolumn{7}{|c|}{$P_{h v}=50 \%$} \\
\hline \multirow{3}{*}{$\begin{array}{c}K U \\
(\text { graus } / \mathrm{km})\end{array}$} & \multicolumn{6}{|c|}{$R F(\mathrm{~m} / \mathrm{km})$} \\
\hline & \multicolumn{3}{|c|}{$\leq 20$} & \multicolumn{3}{|c|}{$20-40$} \\
\hline & $a$ & $b$ & $R^{2}$ & $a$ & $b$ & $R^{2}$ \\
\hline$\leq 50$ & 63,60 & $-0,508$ & 0,79 & 62,30 & $-0,703$ & 0,82 \\
\hline $100-150$ & - & - & - & 57,54 & $-0,870$ & 0,92 \\
\hline $150-200$ & 58,86 & $-0,661$ & 0,88 & - & - & - \\
\hline
\end{tabular}

\subsubsection{Efeito de faixas adicionais}

vés de um fator aditivo $\triangle A T S_{d, \text { car }}$. Para tanto, foram modelados no CORSIM os segmentos usados para gerar os dados sintéticos sem e com as faixas adicionais e foi determinado o acréscimo de velocidade trazido pela implantação da faixa.

Entre os diversos parâmetros estudados (fluxos direé formado por seções com e sem faixas adicionais. $\mathrm{O}$ impacto das faixas adicionais na $A T S_{d, c a r}$ foi determinado atracionais, $P_{h v}, R F, K U$, declividade, etc.), o comprimento da 
faixa adicional $L$ foi a variável independente que apresentou a maior correlação com os valores de $\triangle A T S_{d, c a r}$. A inclusão de algumas das outras variáveis independentes, apesar de ter correlação com as diferenças de velocidade, não aumentou muito o coeficiente de determinação $R^{2}$ do modelo, com nível de significância de 0,05 . Assim, somente o comprimento $L$ da faixa adicional foi suficiente para estimar as diferenças de velocidades, com significância geral boa $(p<$ $0,05)$.

Assim sendo,ATSpl $l_{\text {,car }}$, a velocidade média de percurso na direção estudada num trecho com faixa adicional de comprimento L é obtida somando-se uma parcela à velocidade média de percurso obtida usando-se a Equação 4 $\left(A T S_{d, c a r}\right)$ e os parâmetros de calibração apropriados (Tabela 3) de tal forma que:

$$
A T S p l_{d, c a r}=A T S_{d, c a r}+0,00589 \cdot L . \quad\left(R^{2}=0,78\right)
$$

Uma vez estimada a velocidade média de percurso para o trecho $\left(A T S_{d, c a r}\right.$ ou $\left.A T S p l_{d, c a r}\right)$ o valor da medida de desempenho pode ser calculado usando-se as Equações $1 \mathrm{ou}$ 2. A seção a seguir discute a adequação das medidas de desempenho estudadas.

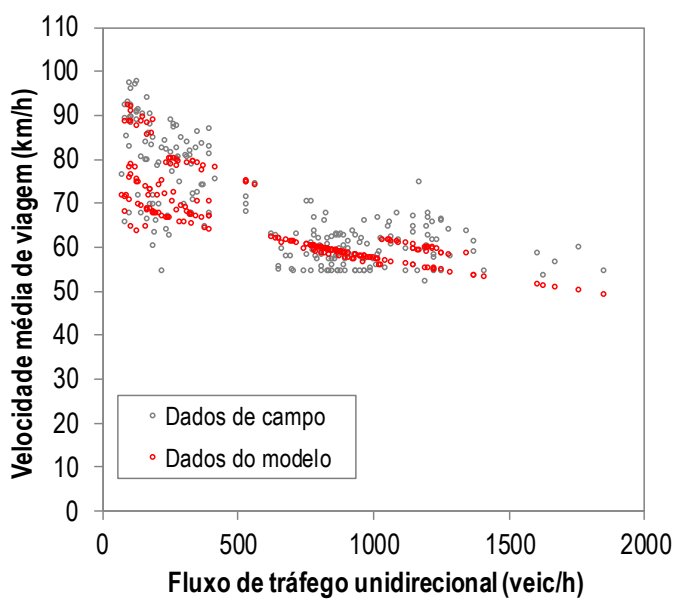

(a)

\section{AVALIAÇÃO DAS MEDIDAS DE DESEMPENHO ESCOLHIDAS}

Uma vez obtidas as relações fluxo-velocidade e os modelos para determinar o impacto da inclusão de faixas adicionais, foi avaliado se os modelos calibrados forneciam valores adequados para a velocidade média de percurso direcional $\left(A T S_{d, c a r}\right.$ ou $\left.A T S p l_{d, c a r}\right)$ e para as medidas de desempenho $P D_{d, c a r}$ e $D_{d . c a r}$, que podem ser calculadas através das Equações 1 e 2. A Figura 5 mostra os resultados dessa análise, na qual foi utilizada a metade das correntes de tráfego obtida das filmagens, conforme explicado na seção 2.

Percebe-se que a dispersão das três medidas de desempenho, tanto para os valores observados como para os gerados pelos modelos, é bem distinta. Para a densidade, a dispersão é pequena; para a velocidade média de percurso é maior; e, para o atraso percentual, é muito grande. De qualquer forma, analisando-se os gráficos, nota-se que os modelos parecem ter fornecidos valores que, com exceção do atraso percentual, reproduzem bem as observações de campo.

A avaliação do poder preditivo dos modelos propostos foi realizada através de funções para determinação da qualidade do ajustamento (goodness of fit): (i) o erro normalizado médio $(M N E)$, que indica um erro sistemático; (ii)

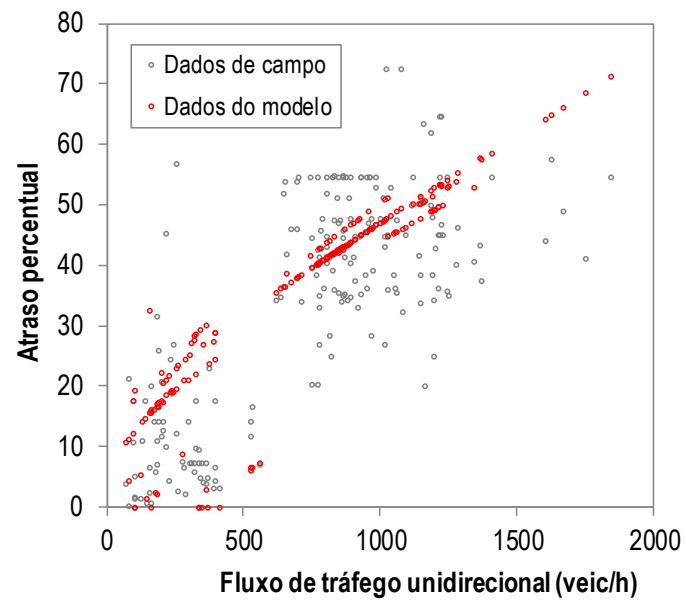

(b)

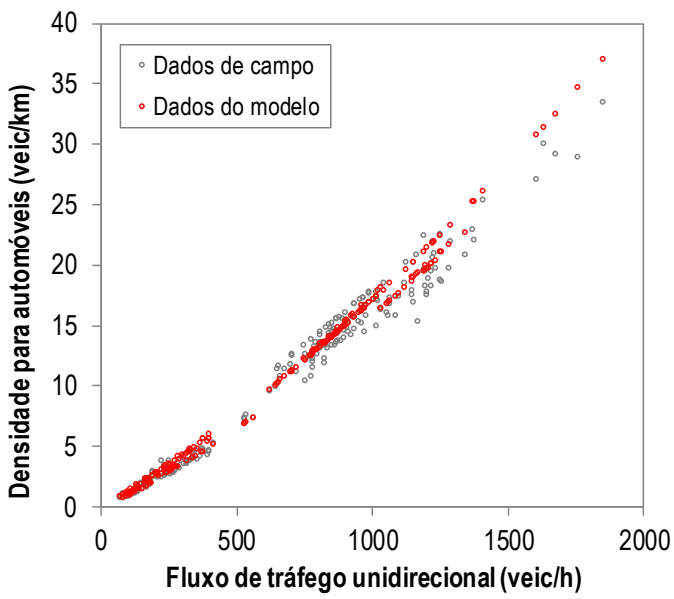

(c)

Figura 5: Medidas de desempenho obtidas em campo e obtidas dos modelos de tráfego para parte dos dados coletados com filmadoras: (a) velocidade média de percurso; (b) atraso percentual; e (c) densidade para automóveis 
o erro normalizado absoluto médio (MANE), que indica um erro médio em termos absolutos; (iii) a raiz quadrada do erro normalizado quadrático médio ( $R M S N E)$, que penaliza erros grandes; e (iv) o coeficiente de correlação $(r)$, que reflete tanto o desvio em torno da média como em função da dispersão dos valores gerados pelos modelos, indicando proporcionalidade entre o valor previsto e o observado, e, não necessariamente, igualdade (Hollander e Liu, 2008).

A Tabela 4 mostra os valores encontrados para a qualidade do ajustamento, sendo que $M N E$ e $M A N E$ podem ser expressos em porcentagem. Das três medidas de desempenho consideradas neste estudo, a densidade $D_{d, \text { car }}$ foi a que obteve os melhores resultados, com $r$ de 0,99 e valores baixos para as demais funções de ajustamento usadas. Para $A T S_{d, c a r}$ e $P D_{d, c a r}$, o coeficiente de correlação foi semelhante, em torno de 0,80 . A qualidade do ajustamento para a função que prediz o atraso percentual (medido através de $M N E, M A N E$ e RMSNE) foi inaceitavelmente baixa, o que pode ser observado no gráfico da Figura 5. A função que estima a velocidade média de percurso mostrou também ter um ajuste de boa qualidade, similar ao nível encontrado para a densidade dos automóveis, com valores similares, à exceção do coeficiente de correlação $r$.

Tabela 4. Qualidade do ajustamento (goodness of fit) para as medidas de desempenho estudadas

\begin{tabular}{lcccc}
\hline \multirow{2}{*}{ Medida de desempenho } & \multicolumn{4}{c}{ Qualidade de ajustamento } \\
\cline { 2 - 5 } & $M N E$ & MANE & RMSNE & $r$ \\
\hline$A T S_{\text {car }}$ & $-3 \%$ & $8 \%$ & 0,10 & 0,82 \\
$P D_{\text {d,car }}$ & $68 \%$ & $92 \%$ & 3,04 & 0,78 \\
$D_{\text {d.car }}$ & $5 \%$ & $8 \%$ & 0,11 & 0,99 \\
\hline
\end{tabular}

Com base nesses resultados, recomenda-se usar, como medida de desempenho para determinar a qualidade de serviço, a velocidade média de percurso e a densidade, ambos para automóveis. Dessa forma, a primeira vai ao encontro do proposto pelo HCM2010 (sendo uma das medidas de desempenho propostas pelo manual), enquanto que a segunda corrobora com o manual alemão HBS2001, que adota $D_{d, c a r}$ como a única medida de desempenho para determinar o nível de serviço em rodovias de pista simples.

\section{CONSIDERAÇÕES FINAIS}

Este trabalho teve como meta avaliar e propor medidas de desempenho que pudessem ser obtidas de relações fluxo-velocidade e que fossem adequadas para descrever a qualidade de serviço em rodovias de pista simples no Brasil. Em vista dos resultados obtidos, conclui-se que duas medidas de desempenho poderiam ser usadas: a velocidade média de percurso dos automóveis e a densidade para automóveis. A primeira, conceitualmente, faz parte do cálculo da segunda. Sendo assim, como a velocidade média de percurso não poderia ser usada sozinha por não retratar o desconforto de viajar atrás de veículos lentos, deve-se utilizar um critério baseado na densidade para automóveis para determinar o nível de serviço em rodovias brasileiras.

Recomenda-se a continuação da análise desses modelos de tráfego com dados de fora de São Paulo e de Minas Gerais. A coleta de novos dados poderia incluir segmentos de estradas com uma maior diversidade geométrica, tanto para gerar dados sintéticos quanto para validar os novos modelos de tráfego. Seria interessante, ainda, investigar o efeito das zonas de ultrapassagens proibidas.

Algumas medidas de desempenho voltadas para rodovias de pista simples não são provenientes de relações fluxo-velocidade. É o caso, por exemplo, da densidade de veículos em pelotões (Van As, 2003), da porcentagem de veículos retidos (Al-Kaisy e Durbin, 2007) e da intensidade do fluxo e da liberdade do fluxo (Cohen e Polus, 2011). Recomenda-se aplicar o mesmo método utilizado neste trabalho (Figura 3) para avaliar essas medidas de desempenho.

Outra necessidade é a obtenção de um critério para determinar o nível de serviço com base na densidade para automóveis (parte final apresentada no método da Figura 3). Para isso, recomenda-se considerar a opinião dos usuários, algo que exige uma pesquisa voltada exclusivamente para tratar essa questão. Com a obtenção do critério, é possível determinar qual o impacto que o uso do novo método para calcular o nível de serviço proporcionaria, em relação ao método proposto pelo HCM2010.

Os resultados encontrados neste trabalho poderiam subsidiar o desenvolvimento de um método voltado para avaliar a qualidade de serviço em rodovias de pista simples brasileiras, o que ajudaria na criação de um Manual de Capacidade Viária brasileiro. A obtenção de um manual brasileiro teria grandes implicações na economia local, como a determinação correta do nível de serviço, usada em várias situações, como o acompanhamento de concessões rodoviárias.

\section{AGRADECIMENTOS}

Os autores agradecem o apoio da FAPESP (Proc. 2010/01809-0) e do CNPq (Proc. 308572/13-0) pelo suporte financeiro sob a forma de bolsas de doutorado e de produtividade em pesquisa. Os autores também agradecem à ARTESP, ao DER-SP e às concessionárias Intervias, Colinas, Renovias, ViaOeste e Rodovias do Tietê pelo apoio nas coletas de dados em campo.

\section{REFERÊNCIAS}

Andrade, G. R. e J. R. Setti (2011) Método para Caracterização e Classificação de Trechos Homogêneos Rodoviários. VII Congresso Brasileiro de Rodovias e Concessões, Anais..., Foz do Iguaçu. ABCR.

Al-Kaisy, A. e C. Durbin (2007) Estimating percent time spent following on two-lane highways: field evaluation of new methodologies. Transportation Research Board 2007 Annual Meeting CD-ROM. TRB, National Research Council, Washington, D.C.

Bessa Jr., J. E. (2015) Medidas de desempenho para avaliação da qualidade de serviço em rodovias de pista simples brasileiras. 128 p. Tese (Doutorado). EESC-USP, São Carlos.

Bessa Jr., J. E.; M. C. Posterlli e J. R. Setti (2013) Estimativas da Porcentagem de Tempo Viajando em Pelotões baseadas em dados coletados em campo. Anais do XXVII Congresso de Pesquisa e Ensino em Transportes, ANPET, Belém-PA.

Bessa Jr., J. E. e J. R. Setti (2012) Relações fluxo-velocidade para rodovias de pista simples no Brasil. Anais do XXVI Congresso de Pesquisa e Ensino em Transportes, ANPET, Joinville-SC, CD-ROM. 
Bessa Jr., J. E. e J. R. Setti (2011) Derivation of ATS and PTSF Functions for Two-lane, Rural Highways in Brazil. 6th International Symposium on Highway Capacity and Quality of Service, Procedia Social and Behavioral Sciences, n. 16, p. 282 292, Stockholm, Sweden. DOI: 10.1016/j.sbspro.2011.04.450

Cohen, M. e A. Polus (2011) Estimating percent-time-spentfollowing on two-lane rural highways. Transportation Research Part C, v. 19, p. 1319-1325. DOI: 10.1016/j.trc.2011.03.001

Cunha, A. B. L. N.; J. E. Bessa Jr. e J. R. Setti (2009) Genetic algorithm for the calibration of vehicle performance models of microscopic traffic simulators. Progress in Artificial Intelligence - Proceedings of the 14th Portuguese Conference on Artificial Intelligence, Aveiro, Portugal. Springer-Verlag, v. 5816. p.3-14. DOI: 10.1007/978-3-642-04686-5_1

DNIT (2011) Manual de Gerência de Pavimentos. Ministério dos Transportes, Departamento Nacional de Infra-Estrutura de Transportes, Rio de Janeiro, RJ.

\section{Egami, C. Y. (2006) Adaptação do HCM-2000 para} determinação do nível de serviço em rodovias de pista simples sem faixas adicionais no Brasil. 240p. Tese (Doutorado). EESCUSP, São Carlos.

FGSV (2005) Handbuch zur Bemessung von Strassenverkehrsanlagen 2001 (HBS, German Highway Capacity Manual 2001). Forschungsgesellschaft fur Straßen- und Verkehrswesen (FGSV), Cologne.

Hollander, Y.; Liu, R. (2008) The principles of calibrating traffic microsimulation models. Transportation, Springer Science and Business Media, LLC, v. 35, p. 347-362. Published online: 15 January 2008. DOI: 10.1007/s11116-007-9156-2

Kerali, H. G. R; D. McMullen e J. B. Odoki (2000) Volume two: Applications Guide. The Highway Development and Management Series Collection, ISOHDM Technical Secretariat, School of Civil Engineering, The University of Birmingham.

Laval, J. A. (2006) A macroscopic theory of two-lane rural roads. Transportation Research Part B, v. 40, p. 937-944. DOI: 10.1016/j.trb.2006.03.002

Luttinen, R. T. (2001) Percent Time-Spent-Following as performance measure for two-lane highways. Transportation Research Record 1776, p. 52-59. DOI: 10.3141/1776-07

Maldonado, M. O.; M. Herz e J. Galarraga (2012) Modelación de operación en carreteras argentinas y recomendaciones de ajustes al Manual de Capacidad HCM 2010. Transportes, v. 20, n. 3, p. 51-61. DOI: 10.4237/transportes.v20i3.556

McTrans (2010) CORSIM - User's Guide. TSIS Version 6.2, McTrans, University of Florida.

Mon-Ma, M. L. (2008) Adaptação do HCM-2000 para rodovias de pista simples com faixas adicionais típicas do Estado de São Paulo. 162p. Tese (Doutorado). EESC-USP, São Carlos.

Nunes, D. F.; M. M. P. Ribeiro; R. B. El-Hage; G. Frasnelli e J. L. Fernandes Jr. (2010) Análise econômica da influência de rampas sobre os custos totais da modalidade rodoviária. Coninfra $2010-4^{\circ}$ congresso de infraestrutura de transportes. Anais ..., São Paulo-SP.

Penmetsa, P.; I. Ghosh e S. Chandra (2015) Evaluation of performance measures for two-lane intercity highways under mixed traffic conditions. Journal of Transportation Engineering, ASCE, p. 1-7. DOI: 10.1061/(ASCE)TE.1943-5436.0000787

Pursula, M. (1995) Approximation of percentage time delay with local measurements. Transportation Research Record 1484, TRB, National Research Council, Washington, D.C, p. 58-65.

TRB (2010) Highway Capacity Manual 2010. Transportation Research Board. Washington D.C.

TRB (2000) Highway Capacity Manual 2000. Transportation Research Board. Washington D.C.

Van As, C. (2003) The development of an analysis method for the determination of level of service of two-lane undivided highways in South Africa. Project Summary. South African National Roads Agency Limited.

Yu, Q. e S. Washburn (2009) Operational performance assessment for two-lane highway facilities. Journal of Transportation Engineering, ASCE, v. 135, n. 4, p. 197-205. DOI: 10.1061/(ASCE)0733-947X(2009)135:4(197) 